\title{
DSC and conductivity studies on PVA based proton conducting gel electrolytes
}

\author{
S L AGRAWAL* and ARVIND AWADHIA \\ Department of Physics, A.P.S. University, Rewa 486 003, India
}

MS received 21 May 2004; revised 15 September 2004

\begin{abstract}
An attempt has been made in the present work to prepare polyvinyl alcohol (PVA) based proton conducting gel electrolytes in ammonium thiocyanate $\left(\mathrm{NH}_{4} \mathrm{SCN}\right)$ solution and characterize them. DSC studies affirm the formation of gels along with the presence of partial complexes. The cole-cole plots exhibit maximum ionic conductivity $\left(2.58 \times 10^{-3} \mathrm{~S} \mathrm{~cm}^{-1}\right)$ for gel samples containing $6 \mathrm{wt} \%$ of PVA. The conductivity of gel electrolytes exhibit liquid like nature at low polymer concentrations while the behaviour is seen to be affected by the formation of $\mathrm{PVA}-\mathrm{NH}_{4} \mathrm{SCN}$ complexes upon increase in polymer content beyond $5 \mathrm{wt} \%$. Temperature dependence of ionic conductivity exhibits VTF behaviour.
\end{abstract}

Keywords. Polymer gel electrolytes; ionic conductivity; solvent free polymer electrolytes.

\section{Introduction}

Over the years, polymer electrolytes have emerged as potential ionic materials for application in different electrochemical devices due to some special properties like good electrode-electrolyte contact, ease of preparation in different forms, good mechanical and adhesive properties (MacCallum and Vincent 1987; Gray 1991). However, their ionic conductivity is usually limited by segmental mobility and concentration of charge carriers (Bruce 1995; Shukla and Agrawal 2000a), which has impeded its utility in electrochemical devices like electrochromic devices, solid state batteries and fuel cells. Since, out of both crystalline and amorphous phases present in polymer electrolytes, only the amorphous phase is the high conducting phase (Berthier et al 1983), plasticizers are usually added to enhance the amorphous phase and thus the ionic conductivity value (Kelly et al 1985). An alternative approach to enhance ionic conductivity in recent years had been through incorporation of fillers, leading to formation of composite polymer electrolytes (Wieczorek 1994; Shukla and Agrawal 2000b). However, in these electrolytes, particle size and concentration of insulating matrix decide their conductivity. Another category of polymer electrolyte with high ionic conductivity is polymeric gel electrolyte (Koksbang et al 1994; Chandra et al 2000; Sekhon 2001; Caillon-Caravanier et al 2003), wherein polymer is reported to be not taking part in conduction process rather acting only as stiffener. In contrast to solid polymer electrolytes, gel electrolytes provide liquid like medium for ion transport and thus have the capability to improve ionic conductivity by an order of magnitude approaching the conductivity of

\footnotetext{
*Author for correspondence
}

liquid electrolytes (Berthier et al 1983; Kelly et al 1985; Wieczorek et al 1994). On account of these interesting properties, gel electrolytes have attained precedence over conventional polymer electrolytes. In recent years, few reported gel electrolytes are polymer network of polymethyl methacrylate (PMMA), polyacrylonitrile (PAN), polyvinylidene fluoride $(\mathrm{PVdF})$ and polyethylene oxide (PEO) (Koksbang et al 1994; Caillon-Caravanier et al 2003). According to available literature, most of the work reported concern lithium salts like $\mathrm{LiClO}_{4}, \mathrm{Li}\left(\mathrm{CF}_{3} \mathrm{SO}_{3}\right)$ etc essentially for lithium based polymer batteries (Feuillade and Perche 1975; Koksbang et al 1994). Proton conductors are equally important class of electrolytes due to their application in fuel cells, ECDs and other devices (Colomban 1992). Within the family of proton conductors, work on PEO based gel electrolytes containing strong acids like $\mathrm{H}_{3} \mathrm{PO}_{4}, \mathrm{H}_{2} \mathrm{SO}_{4}$ and $\mathrm{HCl}$ (Radhucha et al 1996) and PMMA based gel electrolytes containing benzoic and dicarboxylic acids (Chandra et al 2000) have been reported in recent years. According to available literature, polyvinyl alcohol (PVA) is an yet another important polymer network for preparation of proton conducting gel electrolytes on account of its solvent swollen characteristics and wide temperature window (Shukla and Agrawal 2000a). Thus, in the present investigation, an attempt has been made to synthesize PVA based gel electrolytes with ammonium salt for proton conduction and characterize them.

\section{Experimental}

\subsection{Synthesis}

In the present investigation, polyvinyl alcohol (average molecular weight, 124000-186000), Aldrich make, am- 
monium thiocyanate $\left(\mathrm{NH}_{4} \mathrm{SCN}\right), \mathrm{AR}$ grade, sd fine chem make, and aprotic solvent dimethyl sulfoxide (DMSO) AR grade, sd fine chem make, were used for synthesis of proton conducting gels. PVA was dispersed in different stoichiometric ratios in $0.2 \mathrm{M}$ solution of $\mathrm{NH}_{4} \mathrm{SCN}$ electrolyte prepared in DMSO. Addition of polymer to liquid electrolyte was carried out very slowly for formation of homogeneous gel. After syneresis, gels in form of thick films were taken out and dried at room temperature to obtain stable gel electrolytes.

\subsection{DSC studies}

Differential scanning calorimetry of pristine materials as well as few dried gel electrolyte samples were performed on Dupont series 2000 thermal analyser, USA, with a scanning rate of $5 \mathrm{~K} \cdot \mathrm{min}^{-1}$ in nitrogen purged cell in the temperature range $298-523 \mathrm{~K}$ to assess their thermal behaviour and morphology.

\subsection{Electrical measurements}

The gel electrolytes were sandwiched between two platinum electrodes and electrical conductivity measurements were performed on an Hoiki make LCR meter (Model 3520) in the frequency range $40 \mathrm{~Hz}-100 \mathrm{kHz}$ at various temperatures ranging between $287 \mathrm{~K}$ and $373 \mathrm{~K}$. Dielectric spectroscopic measurements were also carried out on gel samples using LCR meter at ambient temperature to understand its role in ionic conductivity.

\section{Results and discussion}

\subsection{DSC studies}

It is well established that crystalline and amorphous phases in variable amount co-exist in most of the polymeric materials (Tager 1978). PVA is one of the partially crystalline polymers exhibiting both the glass transition temperature, $T_{\mathrm{g}}$ (characteristic of amorphous phase) and melting isotherm, $T_{\mathrm{m}}$ (characteristic of crystalline phase), supporting the above argument. This was also evidenced in the DSC thermograms of figure 1 , which represent the thermogram for pure PVA and $\mathrm{NH}_{4} \mathrm{SCN}$ along with a few synthesized gel electrolytes. The glass transition temperature around $363 \mathrm{~K}$ and melting temperature around $489 \mathrm{~K}$ obtained for pure PVA (curve b) are in close proximity to the results of Gong and Cai (1989), Shukla and Agrawal (2000a) and since polymer gel electrolytes are characterized by salt solution (liquid electrolyte) trapped within the polymeric network, thermal behaviour of gel electrolytes is expected to be affected on account of interaction between components and is evidenced in the present study as well. When $2 \mathrm{wt} \%$ of polymer (PVA) was added to $0.2 \mathrm{M}$ ammonium thiocyanate electrolyte to form the gel, the melting isotherm as well as glass transition temperature of PVA did not change (curve c). In addition to these two transitions, a broad melting isotherm (around $417 \mathrm{~K}$ ) corresponding to ammonium thiocyanate (curve a) was noticed. The absence of any other isotherm suggests the existence of two-phase material (PVA and $\mathrm{NH}_{4} \mathrm{SCN}$ electrolyte) with no interaction within components. When the polymer content was enhanced to $4 \mathrm{wt} \%$ in the electrolyte, interaction of PVA with salt was apparent as evidenced

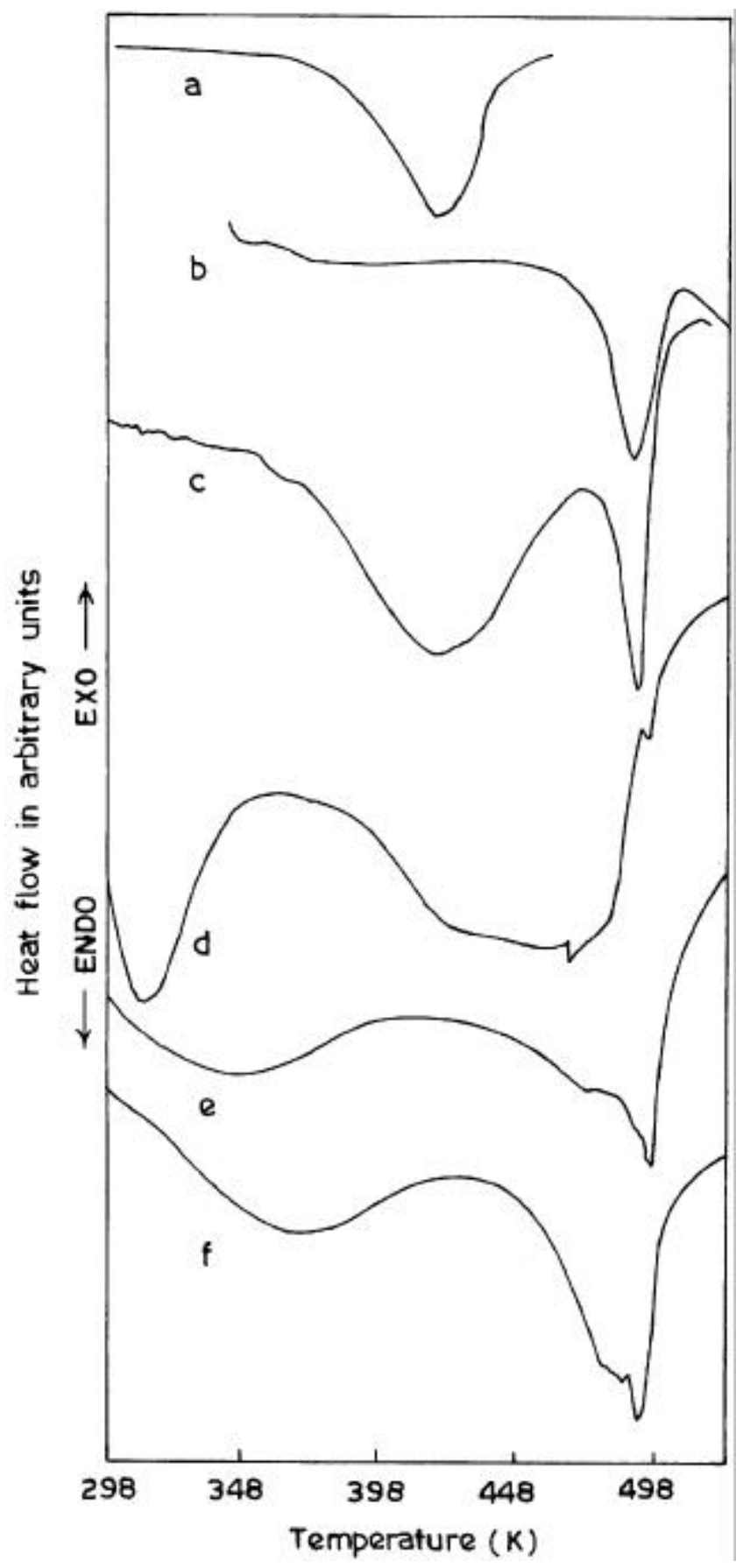

Figure 1. DSC thermogram of (a) $\mathrm{NH}_{4} \mathrm{SCN}$, (b) pure PVA and gel samples containing (c) $2 \mathrm{wt} \%$, (d) $4 \mathrm{wt} \%$, (e) $5.5 \mathrm{wt} \%$ and (f) $8 \mathrm{wt} \%$ PVA. 
action of PVA with salt was apparent as evidenced from the existence of broad endothermic transition (curve d) in the temperature range 423-483 K. PVA is known to form solvent free proton conducting complexes with ammonium salts and acids (Gong and Cai 1989; Gupta and Singh 1996; Shukla and Agrawal 2000a). Thus the observed broadness can be assigned to co-existence of uncomplexed electrolyte $\left(\mathrm{NH}_{4} \mathrm{SCN}\right), \mathrm{PVA}^{-} \mathrm{NH}_{4} \mathrm{SCN}$ complex and uncomplexed PVA. The presence of partially complexed PVA- $\mathrm{NH}_{4} \mathrm{SCN}$ was also evidenced in the DSC thermogram of gel films containing higher polymer content (5.5 wt $\%$ and $8 \mathrm{wt} \%$ PVA viz. curves e $\&$ f). Close observation of DSC thermogram (curve d) indicates a strong endothermic transition around $308 \mathrm{~K}$. The occurrence of this isotherm is probably on account of interaction of methyl group of DMSO with $\mathrm{OH}$ group of the polymer PVA. Dimethyl sulfoxide is known to be a good plasticizing agent with several polymers (Deepa et al 2001) and hence the above possibility cannot be ruled out in the present case. Further, this isotherm was noticed to shift towards higher temperature with increase of polymer concentration in the gel electrolyte. Such a shift might be possibly due to enhancement in attachment of methyl group of DMSO with polar group of PVA leading to rapid gelification. This was also experienced during synthesis i.e. upon enhancement in PVA content, rate of gelification was faster.

\subsection{Conductivity studies}

Figure 2 depicts the variation of ionic conductivity of synthesized gel electrolytes as a function of polymer in the gel electrolytes. The conductivity of liquid electrolyte was found to be $1.5 \times 10^{-2} \mathrm{~S} \mathrm{~cm}^{-1}$. Dimethyl sulfoxide is a highly aprotic solvent and ammonium thiocyanate is

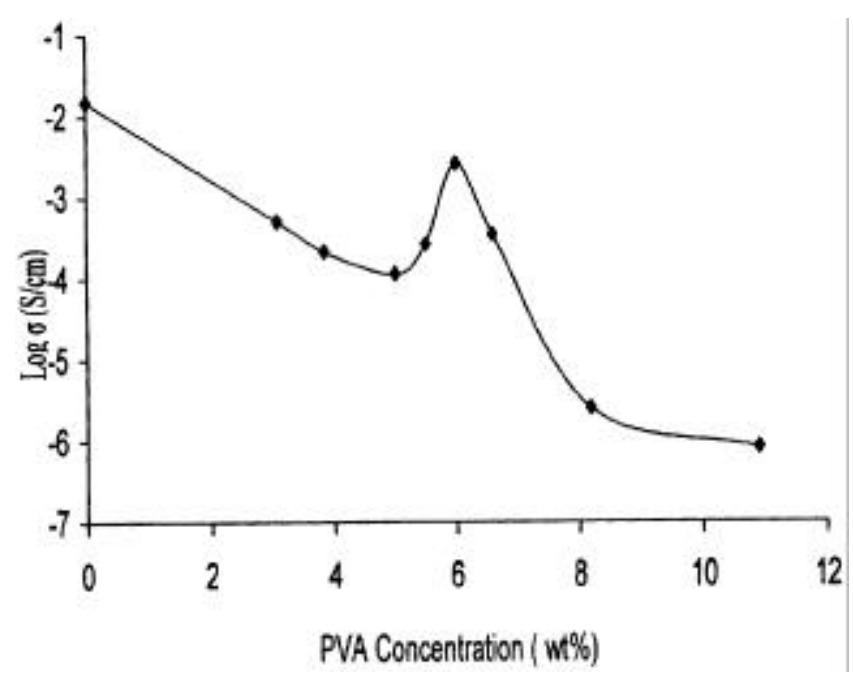

Figure 2. Variation of conductivity as a function of polymer concentration. likely to dissociate into respective ions and behave as liquid electrolyte (Deepa et al 2001) as reflected from high conductivity value. Further the temperature dependence of conductivity ascertains this point of view which shall be discussed later on. When polymer was added to this liquid electrolyte to form gels, the conductivity was initially seen to drop down significantly till $5 \mathrm{wt} \%$ of polymer in the gel. Normally ionic conductivity of electrolyte depends on both the charge carrier concentration, $n$, and carrier mobility, $\mu$, as described by the relation

$$
\sigma=n \cdot q \cdot \mu
$$

with $q$ representing the charge of mobile carrier. Here charge carrier concentration, $n$, depends upon the dissociation energy, $U$, involved and dielectric constant, $\varepsilon$, as

$$
n=n_{0} \exp ^{-U / \varepsilon k T}
$$

where $k$ is the Boltzmann constant and $T$ the absolute temperature. Upon addition of PVA in the electrolyte the dielectric constant $\varepsilon$ was experimentally found to decrease during dielectric spectroscopy measurements (table 1). This means that the decrease in $\varepsilon$ would lead to decrease in value of $n$. Further, addition of PVA also enhances the viscosity of the system which causes a fall in mobility of charge carriers. Both these factors coupled together appear to cause a significant fall in ionic conductivity of gel electrolytes in accordance with (1).

Beyond $5 \mathrm{wt} \%$ of PVA a small increase in ionic conductivity was noticed in the present work. Such an increasing nature of ionic conductivity in gel electrolytes have been reported earlier in case of PMMA based gel electrolytes though at a lower concentration of polymer (Grillone et al 1999; Chandra et al 2000). Chandra et al (2000) tried to explain this rise in ionic conductivity with the help of breathing chain model, wherein they showed enhancement in the charge carrier concentration as a result of ion dissociation caused by folding and unfolding of polymeric chain. In this model, they considered polymeric gel comprising of dissociated ions, ion pairs in the solvent and polymeric chains which may have folded and unfolded or partially folded. The same picture can be visualized here to explain our results i.e. dissociation of ions by local pressure fluctuation leading to increase in the value of $n$. Another plausible reason for increase in ionic conductivity might be due to complexation of $\mathrm{NH}_{4} \mathrm{SCN}$ with PVA as is evidenced from DSC thermogram (figure 1) as well as from earlier reports on formation of solvent free PVA- $\mathrm{NH}_{4} \mathrm{SCN}$ complexes (Shukla and Agrawal 2000a). The two opposing factors i.e. increase in conductivity (i) due to complexation and (ii) due to increase in charge carrier concentration on one hand and fall in conductivity due to increase in viscosity on the other try to counter balance. Consequently, an increase in conductivity till $6 \mathrm{wt} \%$ of polymer in the gel was observed. Beyond this concentration viscosity of the system increases significantly and therefore there is a considerable fall in conductivity as observed in figure 2 . 
Table 1. Dielectric values of gel samples and pristine materials.

\begin{tabular}{|c|c|c|c|c|c|c|}
\hline \multirow[b]{3}{*}{ Frequency } & \multicolumn{6}{|c|}{ Dielectric constant, $\varepsilon$, of samples* } \\
\hline & \multirow[b]{2}{*}{ DMSO } & \multirow{2}{*}{$\begin{array}{l}\text { Liquid } \\
\text { electrolyte }^{\#}\end{array}$} & \multicolumn{4}{|c|}{ Weight per cent of PVA in gel } \\
\hline & & & $2 \mathrm{wt} \%$ & $5 \mathrm{wt} \%$ & $6.6 \mathrm{wt} \%$ & $8 \mathrm{wt} \%$ \\
\hline $500 \mathrm{~Hz}$ & 15 & $10771 \cdot 7$ & $200 \cdot 6$ & 58 & $250 \cdot 3$ & 1.4 \\
\hline $1 \mathrm{kHz}$ & 7.05 & $3341 \cdot 8$ & $134 \cdot 2$ & 31 & $117 \cdot 1$ & 0.7 \\
\hline $10 \mathrm{kHz}$ & $0 \cdot 7$ & $61 \cdot 8$ & $17 \cdot 5$ & $2 \cdot 3$ & $4 \cdot 8$ & 0.08 \\
\hline
\end{tabular}

${ }^{\#} 0.2$ Molar solution of $\mathrm{NH}_{4} \mathrm{SCN}$ in $\mathrm{DMSO} ; * \varepsilon$ correspond to values after division by $10^{3}$.

\subsection{Temperature dependence of conductivity}

Figure 3 shows the temperature dependence of conductivity of $0 \cdot 2 \mathrm{M} \mathrm{NH}_{4} \mathrm{SCN}$ electrolyte along with gel electrolytes containing different wt $\%$ of PVA in the gel. The conductivity of liquid electrolyte is seen to change very slowly with temperature (curve a). Such a behaviour is obvious because dissociation in liquid is almost complete and as a consequence increase in concentration of mobile ions with temperature is unlikely. The gel electrolytes appear to follow a behaviour similar to that of liquid electrolytes, particularly, at lower concentrations of the polymer in the gel. Though the variation of conductivity with temperature for liquid electrolyte is different from that of gel electrolytes, the rate of fall in conductivity with $1 / T$ in the two cases is gradual and within an order of magnitude. This feature is in contrast to PVA- $\mathrm{NH}_{4} \mathrm{SCN}$ solvent free complexes where two arrhenius regions separated by non-linear variation has been reported (Shukla and Agrawal 1996). Observed difference in slopes of gel electrolytes and liquid electrolyte can be attributed to partial complexation of polymer and salt as also evidenced in DSC thermograms. Similar behaviour has been reported for PMMA and PVdF based gel electrolytes (Chandra et al 2000; Sekhon 2001; Kim et al 2002; Kumar and Saikia 2002). Critical examination of the curves (b-g) indicate that ionic conduction of gel electrolytes can best be described by VTF relation (Gray 1991)

$$
\sigma=\sigma_{0} T^{1 / 2} \exp \left(-B /\left(T-T_{0}\right)\right)
$$

where all the terms carry their usual meanings. Besides exhibiting VTF behaviour the temperature dependence of conductivity also depicts significant variation for the gel films containing higher wt $\%$ of PVA i.e. beyond $6.6 \mathrm{wt} \%$ of PVA. This is on account of formation of PVA- $\mathrm{NH}_{4} \mathrm{SCN}$ complexes whose evidence was seen in DSC thermograms.

\section{Conclusions}

Gel electrolytes prepared by addition of PVA to $0 \cdot 2 \mathrm{M}$ $\mathrm{NH}_{4} \mathrm{SCN}$ solution in DMSO have been thermally and electrically characterized in the present work. The gel films appear to behave like liquid electrolyte at lower polymer

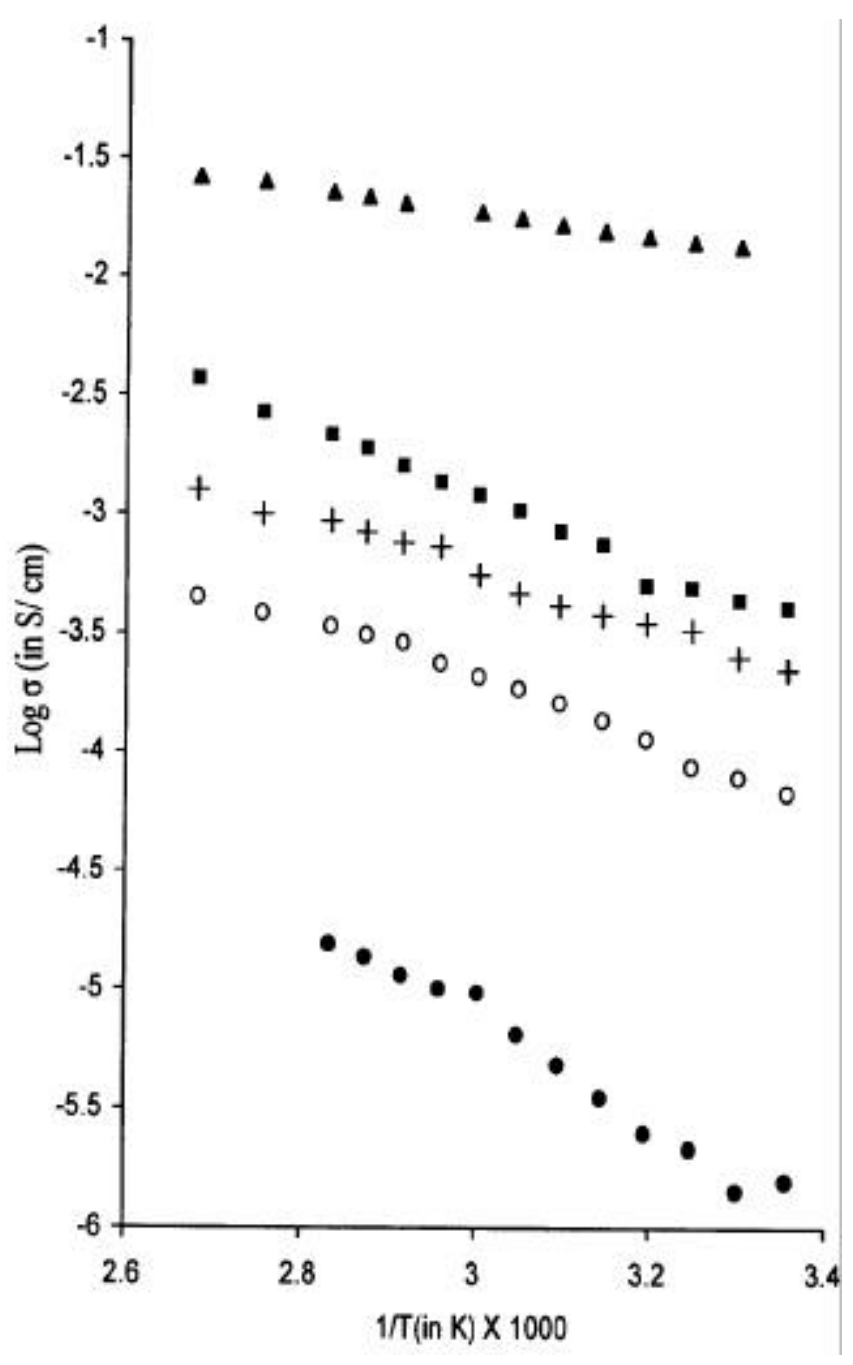

Figure 3. Conductivity as a function of temperature for liquid electrolyte $(\mathbf{\Delta})$ and gels containing $3 \mathrm{wt} \%(\mathbf{\square}), 5 \mathrm{wt} \%$ (O), $6.6 \mathrm{wt} \%(+)$ and $8 \mathrm{wt} \%(\bullet)$ PVA.

content in gel. Gels of higher concentration of polymer shows the presence of PVA- $\mathrm{NH}_{4} \mathrm{SCN}$ complex and conductivity is seen to be influenced by the presence of this complex. The conductivity behaviour can best be described by the breathing chain model in conjunction with 
the properties of $\mathrm{PVA}-\mathrm{NH}_{4} \mathrm{SCN}$ complex. Temperature dependence of ionic conductivity of gel films reflects VTF behaviour.

\section{Acknowledgements}

Authors are thankful to M/s Birla Ericsson Optical Ltd., Rewa, for providing DSC facility. They are also grateful to Prof. S Chandra, Department of Physics, BHU, Varanasi, for stimulated discussions.

\section{References}

Berthier C, Gorecki W, Minier M, Armand M B, Chabango J M and Rigaud P 1983 Solid State Ionics 1191

Bruce P G (ed.) 1995 Solid state electrochemistry (Cambridge: Cambridge University Press)

Caillon-Caravanier M, Claude-Montigny B, Lemordant D and Bosser G 2003 Solid State Ionics 156113

Chandra S, Sekhon S S and Arora N 2000 Ionics 6112

Colomban P (ed.) 1992 Proton conductors: solids, membrane and gels-materials and devices (Cambridge: Cambridge University Press)

Deepa M, Sharma N, Varshney P, Chandra R and Agnihotry S A 2001 in Ion conducting materials: Theory and applications (eds) A R Kulkarni and P Gopalan (New Delhi: Narosa Publishing House) p. 74

Feuillade G and Perche Ph 1975 J. Appl. Electrochem. 563

Gong K C and Cai H S 1989 Solid state ionics (eds) G Nazri, R A Huggins and D F Shriver (Pittsburgh: Materials Research Society) Vol. 135, p. 377
Gray F M 1991 Solid polymer electrolytes: Fundamentals and technological applications (New York: VCH)

Grillone A M, Panero S, Retamal B A and Scrosati B 1999 J. Electrochem. Soc. 14627

Gupta P N and Singh K P 1996 Solid State Ionics 86-88 319

Kelly I E, Owen J R and Steele B C H 1985 J. Power Sources 1413

Kim H S, Shin J H, Choi G Y, Moon S I and Kim S P 2002 in Proceedings of seventh international symposium on advances in electrochemical science \& technology (ed.) M Raghvan (Karaikudi: SAEST), Vol. 1 p. A-84

Koksbang R, Oslen I I and Shackle D 1994 Solid State Ionics 69320

Kumar A and Saikia D 2002 Proceedings of seventh international symposium on advances in electrochemical science \& technology (ed.) M Raghvan (Karaikudi: SAEST) Vol. 1 p. D-52

MacCallum J R and Vincent C A (eds) 1987 \& 1989 Polymer electrolyte reviews (London: Elsevier Applied Sciences Publisher) Vols 1 \& 2

Radhucha D, Wieczorek W, Florjanczyk Z and Stevens J R 1996 J. Phys. Chem. 10020126

Sekhon S S 2001 in Ion conducting materials: theory and applications (eds) A R Kulkarni and P Gopalan (New Delhi: Narosa Publishing House) p. 23

Shukla P K and Agrawal S L 1996 Bull. Electrochem. (India) 12732

Shukla P K and Agrawal S L 2000a Indian J. Pure \& Appl. Phys. 3853

Shukla P K and Agrawal S L 2000b Ionics 6312

Tager A 1978 Physical chemistry of polymers (Moscow: MIR Publishers)

Wieczorek W, Such K, Florjanczyk Z and Stevens J R 1994 J. Phys. Chem. 986840 